\title{
POLYHEDRICITY OF CONVEX SETS IN SOBOLEV SPACE $H_{0}^{2}(\Omega)$
}

\author{
MURALI RAO AND JAN SOKOXOWSKI
}

\section{Introduction}

We provide results on differential stability of metric projection in Sobolev space $H_{0}^{2}(\Omega)$ onto convex set

$$
K=\left\{f \in H_{0}^{2}(\Omega) \mid f(x) \geq \phi(x), x \in \Omega\right\}
$$

where $\Omega \subset R^{d}$ is open, bounded domain.

We derive the form of tangent cone $T_{K}(f)$ for any element $f \in K$-see Theorem 1. The same argument can be used for convex set

$$
K=\left\{f \in H_{0}^{m}(\Omega) \mid f \geq \phi\right\}, m=2,3, \ldots
$$

where $\phi \in H^{m}(\Omega), \phi<0$ on $\partial \Omega$.

In section 3 we provide necessary and sufficient conditions under which set $K$ is polyhedric [5], [8] at a given point $f \in K$. The question of polyhedricity is addressed here since it implies directional differentiability of metric projection onto $K$ with the explicit form of the differential [5], [8]. We refer the reader to [5], [8] for related results in the Sobolev space $H_{0}^{1}(\Omega)$. Some applications of the differential stability of metric projection onto convex sets in Sobolev spaces are presented in [6], [9]-[18].

We recall some properties of the Sobolev spaces and the notion of capacity [19]. The Sobolev spaces $H_{0}^{1}(\Omega)$ and $H_{0}^{2}(\Omega)$ are the closures of $C_{0}^{\infty}(\Omega)$ with norms

$$
\begin{aligned}
\|\varphi\|_{H_{0}^{1}(\Omega)}^{2} & =\int_{\Omega}|\nabla \varphi|^{2} d x \\
\|\varphi\|_{H_{0}^{2}(\Omega)}^{2} & =\int_{\Omega}|\nabla \varphi|^{2} d x
\end{aligned}
$$

Received March 4, 1991. 
respectively. If $\varphi \in H_{0}^{2}(\Omega)$, from the definition $D^{\alpha} \varphi \in H_{0}^{1}(\Omega)$ for each $\alpha$ with $|\alpha|=1$. Functions in $H_{0}^{1}(\Omega)$ are defined quasi everywhere and are quasi continuous. These notions are made precise below.

The $C_{1}$-capacity of a compact set $F$ is defined as

$$
C_{1}(F)=\inf \left\{\int|\nabla \varphi|^{2} d x: \varphi \geq 1 \text { on } F, 0 \leq \varphi \in C_{0}^{\infty}\left(R^{d}\right)\right\}
$$

similarly $C_{2}$-capacity

$$
C_{2}(F)=\inf \left\{\int|\Delta \varphi|^{2} d x: \varphi \geq 1 \text { on } F, 0 \leq \varphi \in C_{0}^{\infty}\left(R^{d}\right)\right\} .
$$

The capacity of a Borel set is then defined as the supremum of capacities of its compact subsets. A statement holds $C_{i}$-q.e., $i=1,2$, if it holds except for a set of $C_{i}$-capacity zero. With this definition we have the following results:

1. Let $\varphi \in H_{0}^{1}(\Omega)$, and $\left\{\varphi_{n}\right\} \subset C_{0}^{\infty}(\Omega)$ converge to $\varphi$ in $H_{0}^{1}(\Omega)$. Then a subsequence of $\left\{\varphi_{n}\right\}$ converge $C_{1}$-q.e. and this is a representative of $\varphi$.

2. Let $\varphi \in H_{0}^{1}(\Omega)$. Then $\varphi$ has a quasicontinuous representative: There is a representative $\bar{\varphi}$ such that given $\varepsilon>0$, there is an open set $U(\varepsilon)$ of $C_{1}$-capacity less than $\varepsilon$ such that the restriction of $\bar{\varphi}$ to the complement of $U(\varepsilon)$ is continuous.

3. Any two quasi continuous representatives of $\varphi \in H_{0}^{1}(\Omega)$ agree $C_{1}$-q.e.

4. Every set of positive Lebesque measure has positive $C_{1}$-capacity.

We use standard notation throughout the paper [1], [19].

\section{Tangent cone}

We shall consider the metric projection onto the following convex set

$$
K=\left\{f \in H_{0}^{2}(\Omega) \mid f(x) \geq \phi(x), x \in \Omega\right\}
$$

with respect to the scalar product

$$
(y, z)=\int_{\Omega} \Delta y(x) \Delta z(x) d x
$$

We assume that $\phi \in H^{2}(\Omega), \phi(x)<0$ on $\partial \Omega$, therefore set (2.1) is nonempty. The metric projection $z=P_{K} y, y \in H_{0}^{2}(\Omega)$, is given by the unique solution of the following variational inequality 


$$
\begin{gathered}
z \in K: \int_{\Omega} \Delta z(x) \Delta(\varphi-z)(x) d x \geq \int_{\Omega} \Delta y(x) \Delta(\varphi-z)(x) d x \\
\forall \varphi \in K
\end{gathered}
$$

We denote

$$
C_{K}(z)=\left\{\varphi \in H_{0}^{2}(\Omega) \mid \exists t>0 \text { such that } z+t \varphi \in K\right\} .
$$

We derive the form of tangent cone $T_{K}(z)=\operatorname{cl} C_{K}(z)$ for any element $z$ in convex set (2.1).

Theorem 1. For any element $z \in K$, tangent cone $T_{K}(z)$ takes the form

$$
T_{K}(z)=\left\{\varphi \in H_{0}^{2}(\Omega) \mid \varphi(x) \geq 0, C_{2} \text {-q.e. on } \Xi\right\}
$$

where $\Xi=\{x \in \Omega \mid z(x)=\phi(x)\} \subset \Omega$.

Proof of Theorem 1. Note that $C_{K}(z)$ and hence also $T_{K}(z)$ is a convex cone containing all non-negative elements of $H_{0}^{2}(\Omega)$. Let an element $V \in H_{0}^{2}(\Omega)$ be given and suppose that $V \geq 0 C_{2}$-q.e. on $\Xi$. There exists the unique element $\phi_{0} \in$ $T_{K}(z)$ such that

$$
\left\|V-\phi_{0}\right\|_{H_{0}^{2}(\Omega)}^{2}=\inf \left\{\left\|V-\phi_{0}\right\|_{H_{0}^{2}(\Omega)}^{2} \mid \phi \in C_{K}(z)\right\} .
$$

It is easy to see that for any $H_{0}^{2}(\Omega) \ni \phi \geq 0, t \geq 0, \phi_{0}+t \phi \in T_{K}(z)$. Using (2.6) and standard arguments it follows

$$
\left(V-\phi_{0}, \phi\right)_{H_{0}^{2}(\Omega)} \leq 0,0 \leq \phi \in H_{0}^{2}(\Omega)
$$

hence there exists a non-negative Radon measure $\mu$ on $\Omega$ such that

$$
\left(V-\phi_{0}, \phi\right)_{H_{0}^{2}(\Omega)} \leq-\int \phi d \mu, \phi \in C_{0}^{\infty}(\Omega)
$$

This implies in particular that for $\phi \geq 0$

$$
\int \phi d \mu=-\left(V-\phi_{0}, \phi\right)_{H_{0}^{2}(\Omega)} \leq\left\|V-\phi_{0}\right\|_{H_{0}^{2}(\Omega)}\|\phi\|_{H_{0}^{2}(\Omega)} .
$$

So by definition of $C_{2}$-capacity we see $\mu$ cannot charge sets of zero $C_{2}$-capacity. Since the measure may be large near the boundary it is not clear that (2.8) holds for all $\phi \in H_{0}^{2}(\Omega)$. We can circumvent this difficulty by repeated use of a result of L. I. Hedberg: Theorem 3.1 in [7]. First we show that (2.8) holds for any bounded $\phi \in H_{0}^{2}(\Omega)$ which is non-negative and has compact support. Indeed for suit- 
able mollifiers $\rho_{n}, \phi * \rho_{n} \in C_{0}^{\infty}(\Omega)$, have compact support, and tend boundedly pointwise $C_{2}$-q.e. and in $H_{0}^{2}(\Omega)$ to $\phi$. Since $\mu$ is Randon measure we may appeal to Lebesque dominated convergence to finish the claim. In the general case if $0 \leq \phi \in H_{0}^{2}(\Omega)$ by the above theorem of Hedberg, we can select $0 \leq w_{k} \leq 1$, $k=1,2, \ldots$ such that $w_{k} \phi$ has compact support and is in $L^{\infty}$ approximating $\phi$ in $H_{0}^{2}(\Omega)$. In particular $w_{k} \phi$ converges to $\phi C_{2}$-q.e. By $(2.8)$ we have

$$
\int w_{k} \phi d \mu=-\left(V-\phi_{0}, w_{k} \phi\right)_{H_{0}^{2}(\Omega)}
$$

is bounded, so by Fatou Lemma $\phi \in L^{1}(\mu)$. On the other hand $w_{k} \phi \leq \phi$ so domin ated convergence applies

$$
-\int \phi d \mu=\left(V-\phi_{0}, \phi\right)_{H_{0}^{2}(\Omega)}, \quad 0 \leq \phi \in H_{0}^{2}(\Omega) .
$$

Now let $\phi \in C_{0}^{\infty}(\Omega), 0 \leq \phi \leq 1$, then $\phi(z-\phi) \in H_{0}^{2}(\Omega)$. We show that

$$
\phi_{0}+t \phi(z-\phi) \in T_{K}(z),-1 \leq t \leq 1
$$

It is sufficient to show that for any $\varphi \in C_{K}(z)$, it follows $\varphi+t \phi(z-\phi) \in$ $C_{K}(z)$. Now $\varepsilon \varphi+z-\phi \geq$ in $\Omega$ for some $\varepsilon>0$, hence for $s>0, \frac{s}{1-s}<\varepsilon$ we have

$$
s[\varphi+t \phi(z-\phi)]+z-\phi \geq 0, \text { in } \Omega
$$

since $(1+s t \phi)(z-\phi) \geq(1-s)(z-\phi)$. Using this in (2.6) with $\phi$ replaced by $\phi_{0}+t \phi(z-\phi)$ we obtain

$$
\left(V-\phi_{0}, \phi(z-\phi)\right)_{H_{0}^{2}(\Omega)}=0
$$

which, because $\phi(z-\phi)$ has compact support and belongs to $H_{0}^{2}(\Omega)$ means

$$
\int \phi(z-\phi) d \mu=0
$$

hence

$$
\mu(x: z>\phi)=0
$$

i.e. $\mu$ is concentrated on $\Xi$. Our next step is to show that $\phi_{0}=0 \mu$-a.e. To this end using the fact that $T_{K}(z)$ is a cone and taking $t \phi_{0}$ for $\phi$ in (2.6) we get

$$
\left(V-\phi_{0}, \phi_{0}\right)_{H_{0}^{2}(\Omega)}=0 .
$$


Now we use Hedberg's result once more. Choose $w_{k}, 0 \leq w_{k} \leq 1$ such that $w_{k} \phi_{0}$ has compact support and converges to $\phi_{0}$ in $H_{0}^{2}(\Omega)$. Since $\phi_{0} \geq 0$ on $\Xi$ and $\mu$ is concentrated on $\Xi, w_{k} \phi_{0} \leq \phi_{0} \mu$-a.e. So using the same argument as above we get

$$
0=\left(V-\phi_{0}, \phi_{0}\right)_{H_{0}^{2}(\Omega)}=-\int \phi_{0} d \mu
$$

i.e. that $\phi_{0}=0 \mu$-a.e.

Finally since $\phi_{0}=0 \mu$-a.e and $V \geq 0 C_{2}$-q.e. on $\Xi$ we can repeat the above argument to get

$$
\left(V-\phi_{0}, V-\phi_{0}\right)_{H_{0}^{2}(\Omega)}=-\int\left(V-\phi_{0}\right) d \mu=-\int V d \mu .
$$

But the right hand side is $\leq 0$ because $V \geq 0$, thus $V=\phi_{0}$.

Remark 1. For $d=1,2,3$ proof of Theorem 1 simplifies since by Sobolev embedding theorem $H_{0}^{2}(\Omega) \subset C(\bar{\Omega})$. It is clear that

$$
T_{K}(u) \subset\left\{\varphi \in H_{0}^{2}(\Omega) \mid \varphi(x) \geq 0, \text { on } \Xi\right\}
$$

therefore it is sufficient to show that any element $V(\cdot) \geq 0$ on $\Xi$ actually belongs to $T_{K}(u)$. $\Xi$ is compact, hence there exists $0 \leq \eta \in C_{0}^{\infty}(\Omega), \eta \equiv 1$ on $\Xi$. Since by Sobolev embedding theorem $u, \phi, V \in C(\bar{\Omega})$ therefore for any $\varepsilon>0$ there exists $t>0$ such that

$$
t(V+\varepsilon \eta)+u-\phi \geq 0, \quad \text { in } \Omega .
$$

Thus

$$
V+\varepsilon \eta \in C_{K}(u), \forall \varepsilon>0
$$

and

$$
V+\varepsilon \eta \rightarrow V \text { in } H_{0}^{2}(\Omega) \quad \text { strongly with } \varepsilon \downarrow 0
$$

hence $V \in \overline{C_{K}(u)}=T_{K}(u)$.

\section{Differentiability of metric projection}

We derive a result on the differentiability of metric projection $P_{K}$ in the Hilbert space $H=H_{0}^{2}(\Omega)$ onto convex closed set $K \subset H$ of the form (2.1). Here we assume for the sake of simplicity that $d=1,2,3$, hence by the Sobolev embedding 
theorem it follows that $H^{2}(\Omega) \subset C(\bar{\Omega})$, the latter embedding is compact [1] for bounded domain $\Omega$ with smooth boundary $\partial \Omega$. We use the following notation. For any given element $u \in K$ we denote

$$
C_{K}(u)=\{\phi \in H \mid \exists t>0 \text { such that } u+t \phi \in K\} .
$$

The tangent cone $T_{K}(u)$ to $K$ at $u$ is the closure of set (3.1)

$$
T_{K}(u)=\operatorname{cl}\left(C_{K}(u)\right) .
$$

Let us consider set $K$ defined in section 1 . We shall address the question of polyhedricity of $K$, see Definition 1 below. Let $T_{K}(f)$ be the tangent cone to $K$ at $f$ $\in K$. It is clear that $T_{K}(f)$ is the closure in the space $H_{0}^{2}(\Omega)$ of the convex cone

$$
C_{K}(f)=\left\{v \in H_{0}^{2}(\Omega) \mid \exists t>0 \text { such that } f(x)+t v(x) \geq \phi(x) \text { in } \Omega\right\} .
$$

For a given element $g \in H_{0}^{2}(\Omega)$, such that $f=P_{K}(g)$ let us define the following convex cone in the space $H_{0}^{2}(\Omega)$

$$
S=T_{K}(f) \cap\left[g-P_{K}(g)\right]^{\perp}=T_{K}(f) \cap[f-g]^{\perp} .
$$

Definition 1. The set $K \subset H_{0}^{2}(\Omega)$ is polyhedric at $f \in K$, if for any $g \in$ $H_{0}^{2}(\Omega)$ such that $f=P_{K} g$ it follows

$$
T_{K}(f) \cap[f-g]^{\perp}=\operatorname{cl}\left(C_{K}(f) \cap[f-g]^{\perp}\right)
$$

here $\mathrm{cl}$ stands for the closure.

Remark 2. Let us recall [5], [8] that if condition (3.5) is satisfied for given elements $(f, g) \in H_{0}^{2}(\Omega) \times H_{0}^{2}(\Omega), f=P_{K}(g)$ then for all $h \in H_{0}^{2}(\Omega)$ and for $t>0$ small enough

$$
P_{K}(g+t h)=P_{K} g+t P_{S} h+o(t) .
$$

In such a case the metric projection $P_{K}$ is conically differentiable, in the notation of [8], at $g \in H_{0}^{2}(\Omega)$. It turns out that condition (3.5) is satisfied if and only if the support of non-negative Radon measure defined below by (3.9) is admissible in the following way.

Definition 2. Compact $F$ is admissible if for any element $\varphi \in H_{0}^{2}(\Omega), \varphi=0$ on $F$ implies $\varphi \in H_{0}^{2}(\Omega \backslash F)$. 
We denote by $B(x, r), x \in R^{d}, r>0$ the ball of radius $r$ and center $x,|A|$ denotes the Lebesque measure of any set $A \subset R^{d}$.

Proposition 1. Let $F \subset \Omega$ be compact and assume that the following holds: for $C_{1}$-quasi every $x \in F$,

$$
|F \cap B(x, r)|>0 \text {. }
$$

Then $F$ is admissible.

Proof of Proposition 1. By Theorem 1.1 in [7] it is sufficient to show the following: let $\varphi \in H_{0}^{2}(\Omega)$ and $\varphi=0 C_{2}$-q.e. on $F$. Then $\nabla \varphi=0 C_{1}$-q.e. on $F$. Now $\varphi \in H_{0}^{1}(\Omega)$ so by a standard result, $\nabla \varphi=0$ a.e. on $F$. Since $\varphi \in H_{0}^{2}(\Omega)$, each component of $\nabla \varphi$ belongs to $H_{0}^{1}(\Omega)$ and hence has a finely continuous version [19]. If for $x \in F,|\nabla \varphi|(x)>0$ then in a fine neighborhood of $x$ the same inequality will obtain. Since finely open sets have positive measure, and since $\nabla \varphi=0$ a.e. on $F$, this violates our condition on $F$. Thus $\nabla \varphi=0 C_{1}$-q.e. on $F$.

Denote by $\nu \geq 0$ the Radon measure defined as follows

$$
\int \varphi d \nu=\int_{\Omega} \Delta(g-f) \Delta \varphi d x, \forall \varphi \in C_{0}^{\infty}(\Omega)
$$

THEOREM 2. We have

$$
\begin{gathered}
\operatorname{cl}\left(C_{K}(f) \cap[f-g]^{\perp}\right) \\
=\left\{\varphi \in H_{0}^{2}(\Omega \backslash F) \mid \varphi \geq 0 \text { on } \Xi \backslash \text { spt } \nu\right\}
\end{gathered}
$$

where spt $\nu \subset \Xi$ is compact, spt $\nu$ denotes the support of Radon measure $\nu$.

Proof of Theorem 2. It is clear that

$$
\operatorname{cl}\left(C_{K}(f) \cap[f-g]^{\perp}\right) \subset S=T_{K}(f) \cap[f-g]^{\perp}
$$

and in view of Theorem 1

$$
S=\left\{\varphi \in H_{0}^{2}(\Omega) \mid \varphi=0 \text { on spt } \nu, \varphi \geq 0 \text { on } \Xi \backslash \operatorname{spt} \nu\right\} .
$$

Let us observe that

$$
H^{2}(\Omega) \ni f-\phi \geq 0, \text { and } f-\phi=0 \text { on compact set } \Xi
$$

therefore it can be shown [20] 


$$
\nabla(f-\phi)=0 C_{1} \text {-q.e. on } \Xi \text {. }
$$

Let $\varphi \in C_{K}(f) \cap[f-g]^{\perp}$ then for some $t>0$

$$
t \varphi+f-\phi \geq 0 \text { on } \Omega \text {, and } \varphi=0 \text { q.e. on } \operatorname{spt} \nu \text {. }
$$

It follows that $\nabla[t \varphi+f-\phi]=0 C_{1}$-q.e. on spt $\nu$ i.e. that $\nabla \varphi=0 C_{1}$-q.e. on spt $\nu$. Clearly the same conclusion obtains for any element in $\operatorname{cl}\left(C_{K}(f) \cap\right.$ $[f-g]^{\perp}$ ) therefore

$$
\operatorname{cl}\left(C_{K}(f) \cap[f-g]^{\perp}\right) \subset H_{0}^{2}(\Omega \backslash \operatorname{spt} \nu) .
$$

Now we can use the same argument as in the proof of Theorem 1 to show that if $V$ is an arbitrary element in set

$$
\left\{\varphi \in H_{0}^{2}(\Omega \backslash \operatorname{spt} \nu) \mid \varphi \geq 0 \text { on } \Xi\right\}
$$

and $\varphi_{0}$ denotes the projection of $V$ onto $\operatorname{cl}\left(C_{K}(f) \cap[f-g]^{\perp}\right)$ then $V=\varphi_{0}$. Thus

$$
\operatorname{cl}\left(C_{K}(f) \cap[f-g]^{\perp}\right)=\left\{\varphi \in H_{0}^{2}(\Omega \backslash \text { spt } \nu) \mid \varphi \geq 0 \text { on spt } \nu\right\} .
$$

Theorem 3. Set $K$ is polyhedric at $f \in K$ if and only if $C_{1}(\Xi)=0$, where $\Xi=\{x \in \Omega \mid f(x)=\phi(x)\}$.

Proof. We show that in (3.9) we can have any nonnegative Radon measure $\nu \in H^{-2}(\Omega)$ with spt $\nu \subset \Xi$. Let such $\nu \geq 0$ be given. Let $g \in H_{0}^{2}(\Omega)$ satisfy

$$
\int_{\Omega} \Delta g \Delta \varphi d x=\int_{\Omega} \Delta f \Delta \varphi d x-\int \varphi d \nu, \forall \varphi \in H_{0}^{2}(\Omega)
$$

We have $f=P_{K} g$. To see it let us observe that

$$
\int \varphi d \nu \geq 0, \forall \varphi \in T_{K}(f)
$$

since $\eta-f \in T_{K}(f), \forall \eta \in K$ it follows

$$
\int(\eta-f) d \nu \geq 0, \forall \eta \in K
$$

hence

$$
\int(\eta-f) d \nu=\int_{\Omega} \Delta(f-g) \Delta(\eta-f) d x \geq 0, \forall \eta \in K
$$

which shows that $f=P_{K} g$. Therefore condition (3.5) can be satisfied if and only if 
$C_{1}(\Xi)=0$

Corollary 1. Assume that $F=\operatorname{spt} \nu$ is admissible then (3.5) and (3.6) hold, where cone $S$ is defined by (3.12).

\section{REFERENCES}

[1] Adams, R. A., Sobolev Spaces, Academic Press, New York (1975).

[2] Ancona, A., Une propriete des espaces de Sobolev, C. R. Acad. Sc. Paris, t. 292, Serie I. 477-480.

[3] Brezis, H. and Browder, F., Some properties of higher order Sobolev spaces, J. Math. Pures Appl., 61 (1982), 245-259.

[4] Coffman, C. V. and Grover, C. L., Obtuse cones in Hilbert spaces and applications to partial differential equations, J. Funct. Anal., 35 (1980), 369-396.

[5] Haraux, A., How to differentiate the projection on a convex set in Hilbert space, Some applications to variational inequalities, J. Math. Soc. Japan, 29 (1977), $615-631$.

[ 6 ] Haug, E. J. and Cea, J. (EDs.), Optimization of Distributed Parameter Structures, Sijthoff and Noordhoff, Alpen aan den Rijn, The Netherlands, (1981).

[ 7 ] Hedberg, L. I., Spectral synthesis in Sobolev spaces, and uniqueness of solutions of Dirichlet problem, Acta Math., 147 (1981), 237-264.

[ 8 ] Mignot, F., Controle dans les inequations variationelles elliptiques, J. Funct. Anal., 22 (1976), 25-39.

[9] Rao, M. and Sokolowski, J., Sensitivity of unilateral problems in $H_{0}^{2}(\Omega)$ and applications, to appear.

[10] - Shape sensitivity analysis of state constrained optimal control problems for distributed parameter systems, Lecture Notes in Control and Information Sciences, Vol. 114, Springer Veriag, (1989), 236-245.

[11] - Differential stability of solutions to parametric optimization problems, to appear.

[12] Sokołowski, J., Differential stability of solutions to constrained optimization problems, Appl. Math. Optim., 13 (1985), 97-115.

[13] - Sensitivity analysis of control constrained optimal control problems for distributed parameter systems, SIAM J. Control Optim., 25 (1987), $1542-1556$.

[14] - Shape sensitivity analysis of boundary optimal control problems for parabolic systems, SIAM J. Control Optim., 26 (1988), 763-787.

[15] - Stability of solutions to shape optimization problems. to appear.

[16] Sokołowski, J. and Zolesio, J. P., Shape sensitivity analysis of unilateral problems. SIAM J. Math. Anal., 18 (1987), 1416-1437.

[17] —, Introduction to Shape Optimization. Shape sensitivity analysis, to appear.

[18] Zarantonello, F. H., Projections on convex sets in Hilbert space and spectral theory, In: Contributions to Nonlinear Functional Analysis, Publ. No. 27, Math. Res. Center. Univ. Wisconsin, Madison, Academic Press, New York, (1971), 237-424. 
[19] Ziemer, P. W., Weakly Differentiable Functions, Springer Verlag, New York, 1989.

[20] - Private communication.

Murali Rao

Department of Mathematics

University of Florida

201 Walker Hall, Gainesville, FL 32611

USA

Jan Sokolowski

Systems Research Institute

Polish Academy of Sciences

ul. Newelska 6, 01-447 Warszawa

Poland 